\title{
6. Escaping social and socioecological traps in complex systems
}

\section{RESILIENCE: THE BRIGHT SIDE AND THE DARK SIDE}

Building on Holling's pioneering work of the 1970s, a dedicated research community has acted vigorously to develop a rich understanding of the concept of resilience and deploy it as the cornerstone of an influential stream of thinking regarding the dynamics of ecological, social, and socioecological systems (Gunderson and Holling 2002). Resilience, in the resultant analytic framework, is a property of systems. Specifically, it is a matter of the "capacity of a social-ecological system to absorb or withstand perturbations and other stressors such that the system remains within the same regime, essentially maintaining its structure and functions" (Resilience Alliance 2020). What distinguishes resilience from older and related concepts such as equilibrium or stability is the idea that a resilient system is able to adapt to changing circumstances without losing its basic character and its capacity to operate successfully. Thus, we can say that a resilient system "learns" by making adjustments needed to allow it to continue to function effectively in the face of pressures that are both internal and external in origin. To elucidate the processes giving rise to resilience, the research community concerned with such matters has devoted considerable energy to developing a dynamic model commonly referred to as the adaptive cycle, a construct originating among ecologists that shows how systems can experience collapse but rebound through processes of reorganization and regrowth needed to adjust to changing circumstances (Holling and Gunderson 2002). Systems that behave in this manner are regarded as adaptive systems.

As applied to socioecological in contrast to purely ecological systems, the idea of resilience has provided not only an analytic framework to be used in enhancing our understanding of the behavior of systems of interest to humans, but also a normative perspective that many find helpful in creating and administering management systems to address a range of policy issues (Walker and Salt 2006). Put simply, resilience is a good thing. Because resilient systems are able "to absorb or withstand perturbations and other stressors," they have 
greater staying power than systems that lack resilience, especially in a world in which biophysical and socioeconomic changes constitute a prominent feature of reality. It follows that we should devote time and energy to thinking about ways to enhance the resilience of major socioecological systems. While key actors may disagree on the pros and cons of specific strategies for pursuing this goal, there is widespread agreement regarding the desirability of taking steps to buttress resilience.

This analytic framework has produced insights of considerable value, especially as we endeavor to maintain the resilience of socioecological systems in the face of major challenges arising today (Folke 2006). That is a substantial achievement. But in this chapter, I endeavor to temper the air of enthusiasm that surrounds the concept of resilience, drawing attention to two complications that deserve serious consideration as we seek to respond to the 21 st century's grand challenges of planetary governance. One complication centers on what I call the dark side of resilience. My purpose in using this phrase is to draw attention to the observation that there are systems that are resilient but generate results that seem undesirable from a variety of normative perspectives; they may even yield outcomes that are worse for all parties concerned than other outcomes that could be achieved if it were possible to break the power of resilience to impede the occurrence of regime shifts or critical transitions. These situations are often characterized as social or socioecological traps (Cross and Guyer 1980; Costanza 1987). As cases like the poverty trap and many traps associated with the tragedy of the commons make clear, systems of this kind may prove highly resilient, resisting even the most determined efforts to escape the force of the relevant traps by instituting reforms designed to alter the dynamics of the systems. In situations of this sort, resilience is a feature of the problem to be solved, rather than a valued property of the system to be enhanced through a variety of supportive measures.

The second complication, cutting across these observations about resilience, arises from the fact that we live in a world of systems that are increasingly complex in the sense that they are characterized by hyperconnectivity, nonlinearity, directional change, and recurrent surprises (Johnson 2009; Levin 1999). As the debate about planetary boundaries has taught us, we need to pay close attention to tipping elements in complex systems and the prospect that seemingly small perturbations can trigger profound changes or bifurcations in apparently resilient systems (Lenton et al. 2008; Rockström et al. 2009). This has produced a lively discussion of thresholds, safe operating spaces, and the need to exercise extreme care regarding human actions that could trigger changes that take us by surprise, producing results frequently thought to be catastrophic. The growing interest in the idea of precaution or, in other words, the virtues of playing it safe is a response to this concern. 
But here, too, we need to think about the flip side of this phenomenon. What I mean by this is that some complex systems feature positive tipping elements giving rise to opportunities to break out of social or socioecological traps (Lenton 2019). In situations of this kind, seemingly modest initiatives can trigger regime shifts, allowing actors to escape the treadmill of grinding poverty or communities to avoid disruption arising from repeated collapses of valuable living resources. In the case of climate change, the danger of passing a tipping point and moving into a more chaotic climate system is a source of extreme concern. But for those struggling to escape from socioecological traps, there is hope in the prospect of locating tipping elements and finding ways to push complex systems toward thresholds where modest additional interventions can trigger processes that weaken the forces of resilience locking actors into dysfunctional situations. Whereas bifurcations in complex systems may loom as dangerous occurrences to be avoided at all costs in the management of functional systems, they can appear as beacons of hope for those caught in dysfunctional traps. Precaution may well be the order of the day in cases where the goal is to preserve a safe operating space for humanity. But bold initiatives offer a better recipe for the pursuit of sustainability in situations where the challenge is to break out of social or socioecological traps.

\section{CRITICAL CLARIFICATIONS}

Before embarking on an effort to unpack these observations, let me comment on several critical issues associated with key features of the concept of resilience. Resilience, in this line of analysis, is a variable. Systems may be more or less resilient, and we want to identify those factors that can be expected to enhance or diminish resilience under specified conditions. But here, too, two complications arise. One has to do with the fact that we lack a precise, much less an operational, metric for measuring resilience or assessing trends in resilience under real-world conditions. This does not mean we are at a complete loss regarding levels or degrees of resilience. For instance, it seems reasonable to say that systems featuring negative feedback mechanisms that kick in when perturbations occur will be more resilient than systems lacking such mechanisms. Research featuring arguments pertaining to levels of resilience have also generated productive debates among members of the research community. A good example is the effort to evaluate the relationship between species diversity and resilience in ecosystems. Nevertheless, the lack of a well-defined metric and a widely accepted set of procedures for measuring trends in levels of resilience is a serious problem for those seeking to develop a body of empirically grounded knowledge about the behavior of socioecological systems, much less a capacity to bring this knowledge to bear as a source of prescriptive advice regarding the management of specific systems. Among other things, 
this limitation encourages us to think in dichotomous rather than continuous terms. A system is resilient until it passes a tipping point, after which it shifts more or less abruptly from one basin of attraction to another (Rockström et al. 2009).

Then, there is the problem of coming to terms with complex causality, a condition that becomes more and more central as we move into a world of complex systems. What this means is that a multiplicity of factors, often interacting with one another, act as determinants of levels of resilience in specific situations. In those rare situations where we have sizable universes of cases to work with, this means that we need to think in terms of the application of procedures like multiple regression, assigning weights to the role of different factors as determinants of variance on the dependent variable. In the more usual situation where we are limited to analyzing a few cases and focusing on regime shifts, it may make more sense to think about conjunctural causation, as articulated by Ragin and others, who have developed the analytic technique known as qualitative comparative analysis (Ragin 1987). The central idea here is that we should search for clusters of forces that, taken together, can explain whether and when dramatic changes occur, including the dichotomy between the occurrence or nonoccurrence of a regime shift or critical transition in a socioecological system.

\section{SOCIAL AND SOCIOECOLOGICAL TRAPS}

A trap is a situation harmful to its victims in which a set of interlocking conditions make it difficult or even impossible for those ensnared in the trap to escape through their own efforts. Of course, we are all familiar with situations in which humans are the trappers and members of other species are the victims. Humans seek to eliminate unwanted rodents through the use of mousetraps; trappers make their livings by establishing and running traplines designed to catch animals like beavers and foxes. In such situations, those who set the traps endeavor to make them as resilient as possible. But the focus of interest in this analysis is on situations in which humans, either as individuals or as members of collectivities, fall into social or sociological traps that they find costly to escape from or that they are unable to extricate themselves from at all, at least in the absence of some effective external intervention. In some cases, the struggle to escape actually increases the resilience of the trap, making escape increasingly difficult. In other cases, the effort to escape one trap triggers the onset of conditions that make victims vulnerable to a related trap.

Resilience in such situations is a matter of the persistence of a trap in the face of changing biophysical or socioeconomic conditions. A resilient trap on this account is one that persists or reasserts itself both in the face of substantial changes in the broader setting and especially in the face of focused efforts 
aimed at eliminating or dismantling the trap itself. In such cases, resilience emerges as a feature of the problem rather than as a feature of systems that enhance their capacity to perform a variety of social functions effectively. We want to devise strategies that lead to the erosion of resilience and improve prospects for exiting social and socioecological traps in contrast to strategies that can enhance the resilience of adaptive systems. To explore the conditions that give rise to traps of this sort and to begin the process of identifying escape mechanisms or measures that mitigate their harmful effects, I draw a distinction between structural traps and collective-action traps.

\section{Structural Traps}

In some societies or social strata within them, a set of interlocking structural conditions serve to impede the efforts of individuals or groups of individuals to enhance their welfare or to improve their circumstances more generally. Consider as an initial example what many think of as the poverty trap. We all know about "rags-to-riches" narratives or Horatio Alger stories in which impoverished individuals "rise from humble backgrounds to lives of middle-class security and comfort through hard work, determination, courage, and honesty" (Wikipedia 2020). But such cases constitute the exception rather than the rule. The fact is that there is a strong correlation between impoverished beginnings and subsequent failure to make good, whether we are looking at the fate of individuals in more or less advanced societies or at the fate of impoverished societies treated as social units. There are several identifiable factors underlying this correlation, including undernourishment, developmental deficiencies, limited access to education, the absence of adequate health care, a scarcity of resources to upgrade infrastructure or invest in new ventures, the degradation of relevant biophysical systems, temptations to engage in illegal activities to alleviate these problems, and so forth. In many cases, the poverty trap is highly resilient. Efforts to intervene to give people a better start in life may benefit some individuals. But they do little to alleviate the poverty trap itself. They may even make things worse for those still caught in the trap by providing an escape route for a few of the best and brightest members of the community, leaving the rest to suffer from the knock-on effects of severe poverty.

Turn next to the case of a socioecological trap arising from the influence of path dependence. During the second half of the 20th century, many Americans fled from harsh conditions prevailing in major cities to seek a better life in newly developing suburbs. Rising incomes during the postwar period made it possible for families to aspire to own their own homes in the suburbs. Developers were happy to oblige by acquiring tracts of land and building new homes to meet the rising demand. Urban and suburban planners were able and 
willing to design infrastructure to accommodate the dispersed and relatively low-density residential pattern associated with suburbanization. The rise of automobile ownership led to the development of transportation systems featuring highways suitable for automobile traffic and the relative neglect of various forms of public transportation. Efforts to assess the benefits and costs of suburbanization are highly controversial. But one thing is clear. From the perspective of major environmental concerns and especially the problem of climate change, suburbanization is a significant part of the problem. It is a major source of the fact that greenhouse gas emissions per capita in the United States are among the highest in the world (Global Carbon Project 2020). What makes this situation a socioecological trap is that suburbanization is in many ways a one-way street. That is, it is hard to envision a strategy that would allow us to reverse the course of this pattern of development. At a minimum, the costs of reversing course, at least on a large scale, are widely regarded as prohibitive. Of course, modest adjustments (installing solar collectors on suburban rooftops) are possible. But such measures do not offer an effective escape route from the trap itself.

Yet another example centers on what I call the authoritarian trap. The key observation in this case is that societies in which authoritarian regimes break down or are forcibly overthrown frequently experience the emergence of new authoritarian regimes following a period of more or less unsuccessful experiments with democratic institutions. Prominent recent examples include the reemergence of authoritarian rule in Russia following the collapse of the Soviet Union and the unfulfilled promise of the Arab Spring in countries like Egypt. In effect, the authoritarian trap is resilient under a variety of conditions as efforts to break out of the trap set in motion processes that lead to a reversion to authoritarian control, albeit with new leadership and perhaps with new mechanisms for asserting control. Why is this the case? It turns out that there are close connections between the ability to resist authoritarian rule and the development of a political culture emphasizing active participation on the part of individual citizens and the creation of political institutions that serve as barriers to the ambitions of powerful leaders. The absence of such conditions in Russia stretching back into czarist times is undoubtedly an important determinant of the lack of effective barriers to authoritarian rule in the 21 st century. Authoritarian rule is typically harmful to the bulk of the population and may prove harmful to the long-term prospects of society as a whole. Nevertheless, there is no shortage of cases in which the authoritarian trap has proven highly resilient.

A final structural case that also offers some insights regarding the prospects for escaping social and socioecological traps involves what many think of as the demographic trap, or the Malthusian trap. The essential idea, as formulated initially by Thomas Malthus at the end of the 18th century, is that any increases 
in food production will stimulate a growth in population, so that society as a whole will revert to a subsistence level over time. This line of thinking has remained influential, especially in times like the post-World War II period during which the Earth's human population has expanded at an extraordinary rate. Yet, on a global scale at least, the trap has not closed (Fogel 2010). A combination of factors, including the clearing of virgin land for farming, the development of improved varieties of plants, the use of chemical fertilizers, and the introduction of pesticides, has allowed food production to increase at a dramatic rate. Although severe famines have occurred in some societies and there are obvious problems regarding the distribution of food within most societies, the rate of growth in global food production is commensurate with the rate of growth in human population. Even so, the concern about the demographic trap has not disappeared. Some ask whether our ingenuity relating to food production has simply delayed the inevitable. Will the cornucopia associated with advances in agricultural technology eventually be depleted as we run into the limits to food production? Equally important are the ecological consequences of the explosion in the population of humans, ranging from rapid increases in greenhouse gas emissions to dramatic losses of biological diversity. This raises a question about whether our efforts to escape the demographic trap have given rise to an even more profound trap involving the long-term sustainability of the human enterprise on a finite planet.

\section{Collective-Action Traps}

Another type of social or socioecological trap arises in situations where actors (ranging from individuals to nation states) pursuing their own interests in interactions with others behave in such a way that the outcomes are harmful or even disastrous for all parties concerned. Traps of this sort involve what Olson describes as the logic of collective action (Olson 1965) and Schelling treats as the interplay between micromotives and macrobehavior (Schelling 1978). Of course, outside intervention may alter the incentives of the relevant actors in such a way as to allow them to escape from collective-action traps. Left to their own devices, however, the actors may find it difficult to devise escape routes; their efforts may even serve to tighten the traps. In other words, some collective-action traps often are characterized by a high level of resilience.

Consider as an initial example a situation involving a common pool resource like an exploitable fish stock that is open to entry on the part of those desiring to engage in harvesting. In the absence of effective restrictions, the harvesters are likely to fall prey to what we have come to know as the tragedy of the commons (Hardin 1968). That is, each harvester will have an incentive to continue fishing knowing that any fish remaining in the stock will be taken by other members of the group. The outcome will be a severe depletion or even 
destruction of the stock that is harmful to the well-being of all those interested in catching and consuming the fish. There is a long history of efforts to introduce restrictions that make it possible to avoid falling into this trap (Webster 2015). Some of these efforts have proven successful, allowing for the achievement of sustainable harvests over indefinite periods of time. What is striking, however, is the resilience of the common pool trap or, in other words, the frequency with which the trap reemerges in one form of another in the wake of various efforts to break out of it. For example, the introduction of open and closed seasons may result in the occurrence of fishing derbies during shorter and shorter openings. The imposition of gear restrictions is often met with increased investments in the types of gear that are permitted in a given fishery. What is more, measures designed to protect fish stocks from severe depletion often result in outcomes that are unappealing in normative terms. There are many instances in which the introduction of individual transferable quotas (ITQs), for example, leads to outcomes in which smallscale coastal fishers are driven out of the industry and levels of economic inequality rise.

Another case centers on the purchase of guns on the part of those who fear for their own safety. In many cases, the result is a spiral in which more and more people acquire weapons and those who have them already upgrade to increasingly powerful weapons, such as assault rifles. Defenders of such behavior argue that people rather than guns kill other people and that self-defense is a reasonable response to such situations. Nevertheless, it is clear that the spread of weapons in a society leads to a rise in the incidence of homicides. Ironically, the evidence suggests that an individual who possesses a gun is more likely to be killed or injured by gunfire than an individual who does not possess a weapon. It turns out that it is difficult - sometimes impossible - to find a way to escape this trap. In the United States today, for example, those who defend the right to bear arms are so influential politically that there is little prospect of imposing serious limitations on the possession and use of firearms through the operation of the political system. And the resultant trap is highly resilient. In fact, there is a tendency for the trap to become increasingly severe as the actions of individual members of society escalate in response to the real or perceived threats arising from the actions of others. In the absence of effective restrictions, the result can be a dynamic in which more and more powerful weapons proliferate within the relevant society over the course of time.

In recent years, students of international relations have devoted considerable attention to what has become known as the Thucydides trap (Allison 2018). The trap refers to a destructive episode in the history of ancient Greece in which the rivalry between Sparta as an aging hegemon and Athens as a rising great power provoked a protracted war during the 5th century bc in which both sides suffered severe losses. Reflecting on this case, analysts have asked whether the mechanism underlying this trap is generic, so that we can expect 
a high probability of destructive warfare breaking out in situations featuring challenges to prevailing political orders in cases where rising powers are perceived to threaten the position of existing hegemons. Some see a dynamic of this sort arising in conjunction with the naval arms race between Great Britain and Germany in the run-up to the outbreak of World War I in 1914. There is a lively discussion today regarding the question of whether a similar dynamic is driving interactions between the United States as an aging hegemon and China as a rising superpower. As critics have observed, it is dangerous to impose simple explanatory models on highly complex historical episodes like the rivalry between Sparta and Athens in ancient Greece, much less to generalize to a range of cases that differ from one another in a number of significant ways. Still, we can see elements of this type of dynamic in the competition between or among leading actors in a variety of social settings ranging from smallscale societies to the global system. The interesting challenge is to identify the conditions leading to the onset of such traps and to determine the extent to which they are resilient in the face of various efforts to escape from them.

A final example, related in some ways to the proliferation of firearms and to the competition among those seeking to achieve dominance within a social system, centers on arms races in which two or more actors compete with one another in an effort to achieve military dominance. As many commentators have noted, arms races often seem to have a life of their own driven by a kind of "mad momentum," consuming extraordinary amounts of resources diverted from other uses and leading to a condition of mutual exhaustion rather than a victory for one or another of the participants (Fairbanks 1985). Most arms races are properly viewed as social traps. But arms races featuring weapons of mass destruction seem particularly pernicious in these terms. Because each participant is apt to have a pronounced fear that falling behind will lead to a situation in which the other side achieves political control, the resultant competition is likely to become particularly intense. Those who study arms races often pose questions about the resilience of these traps, seeking to identify conditions governing the trajectory and intensity of arms races over time. The result is the development of models that allow us to identify in abstract terms conditions under which these action-reaction processes escalate toward some sort of explosion or, conversely, die down toward some sort of stasis (Rapoport 1960; Boulding 1962). It is not easy to map such models onto real-world situations, making it possible to explain or predict the trajectories of actual arms races. But this observation does suggest that it is time to turn to a consideration of the relevance of various features of the overarching systems in which social and socioecological traps emerge and play out. 
Those who focus on resilience and spend time thinking about ways to maintain and even enhance resilience tend to regard the growth of complexity in socioecological systems as a source of challenges. Telecoupling may make a community vulnerable to events occurring far away over which members of the community have little or no control. Consider the case of small coastal communities in remote areas forced to relocate due to the impact of coastal erosion attributable to the global forces of climate change. The prospect that crossing ill-defined thresholds will trigger critical transitions that are difficult to cope with leads to an increasing emphasis on the importance of precaution. The implicit message here is that the dangers of action are greater than the dangers of inaction, though it is apparent that inaction can become a source of problems in some situations. Above all, the frequency of surprises occurring in complex systems can give rise to a pervasive sense of unease or anxiety. Because we are aware that surprises will occur but have little ability to forecast when they will occur or anticipate their consequences, we find it difficult to deploy resources effectively to secure the resilience of social or socioecological systems that we value highly.

Certainly, these are understandable concerns. But I want to argue that the role of complexity emerges in a different light when we focus on the phenomenon of social and socioecological traps and think about what is required to escape or break out of traps that are harmful to all those affected by structural traps or to the specific parties ensnared in collective-action traps. Many (though not all) traps are resilient. The poverty trap, for example, often reasserts itself, even in cases where victims struggle to escape and seem to be making some initial progress in their efforts to climb out of the trap. The confusion or chaos following the collapse of an authoritarian regime can create conditions that are favorable to the rise of some new form of authoritarianism. The tragedy of the commons may reemerge in marine fisheries in some new form, even after the introduction of measures intended to break the power of the interactive decision-making that produces outcomes that are harmful to all. Some technological advance or political spark may reignite an arms race that has been slowed or even stopped by the negotiation of an agreement regarding arms control measures.

An observation that applies to most situations of this sort is that strategies featuring incremental or step-by-step measures to escape social and socioecological traps seldom suffice to break the grip of resilient traps. The poverty trap constitutes a classic example. Interventions may help individual members of a group to escape, gaining an education and rising to a higher stratum in the social order. But the grip of grinding poverty for the bulk of the affected pop- 
ulation typically proves resistant to the influence of a wide range of measures designed to alleviate the impact of poverty at the margin. Similarly, the creation of democratic institutions that look good on paper often fails to suppress the political dynamics leading to the reemergence of authoritarianism. Much the same is true regarding collective-action traps. Specific measures like gear restrictions in marine fisheries or caps on permissible levels of particular types of weapons may weaken the grip of traps in specific instances. But these traps often prove resilient in the sense that they adapt to the influence of the specific measures and reassert their grip over the outcomes arising from the relevant forms of interactive behavior.

What this suggests is a need to consider critical transitions or what are known as bifurcations in thinking about the prospects for escaping from resilient traps (Lenton et al. 2008; Scheffer 2009). Or, to employ the language of researchers who study resilience, there is a need to identify conditions that can trigger a regime shift or cause a jump from one basin of attraction to another. Whereas most students of resilience fear bifurcations and devote substantial time and energy to maintaining a "safe operating space for humanity," those concerned with breaking the grip of social and socioecological traps are likely to treat the possibility of critical transitions and bifurcations as a source of hope. If we can find a way to push a dysfunctional system past a critical threshold or tipping point, modest additional efforts may cause a new dynamic to take hold that leads to fundamental changes in the behavior of the system (Lenton 2019). Of course, it is important to avoid falling prey to naive hopes regarding the nature of such processes. Some traps (e.g., suburbanization) may prove extremely difficult to escape; others (e.g., arms races) can end in explosions that impose high costs on all parties concerned. Still, the role of critical transitions and bifurcations will look very different to those concerned with escaping traps than they do to those concerned with maintaining the capacity of the adaptive cycle to avoid regime shifts.

As a general proposition, it seems accurate to say that the probability of critical transitions and bifurcations occurring rises as systems become more complex. That is why the rise of complex systems may seem promising to those seeking to escape traps, even though they do not deny the difficulty of governing such systems effectively. In this connection, the idea of positive tipping elements comes into focus (Lenton 2019). Most discussions of tipping elements point to forces that can drive systems past thresholds leading to explosions or cascades of change that produce destructive results (at least from a human perspective) and that are difficult or impossible to control. This accounts for the fear that seemingly small occurrences can trigger massive consequences that are harmful to all. But this is not the case with regard to positive tipping elements. The idea here is to locate critical threads that can make all the difference for those struggling to escape from a social or socio- 
ecological trap. Pulling on the right thread may make it possible to release the jaws of the trap in situations where massive campaigns to break out of a trap that fail to focus on the critical issues prove ineffective.

We can identify (at least) three types of (by no means mutually exclusive) tipping elements that are worthy of consideration on the part of those seeking to escape resilient traps. For shorthand purposes, I will describe them as technological, institutional, and behavioral tipping elements. There is a lively debate about the relative merits of strategies featuring reliance on one or another of these elements. It is apparent that there are no magic bullets available to those seeking to escape social and socioecological traps. The success of specific strategies will be affected by a variety of features of specific situations. Nevertheless, a few examples will serve to clarify the differences among approaches associated with each type of tipping element.

The spread of cell phones and the resultant ability to conduct business over the internet has made a striking difference in the efforts of some communities to escape the poverty trap. Half to two-thirds of the residents of some poor African communities, for example, are now able to make a variety of transactions via the internet. This has opened up significant opportunities for those with entrepreneurial skills to launch new businesses that have produced benefits not only for themselves but also for the communities to which they belong. Turning to a very different example, the development of high-resolution satellite observations has made it possible to document compliance with certain types of arms control agreements, without any need to organize intrusive and politically sensitive onsite inspections. Of course, the evidence regarding compliance is not always conclusive, and this can become a problem in situations where only a few violations can make all the difference. Similar observations are in order regarding the development of satellite observations systems that make it possible to determine whether individual ships are in compliance with fisheries regulations. What this means is that technology may reduce but not eliminate the dynamics generating traps or leading to their reemergence following initial efforts to escape.

In some respects, the classic response to social and socioecological traps is to introduce new institutions or governance systems designed to deactivate some key element(s) of the traps (Young 2017). This response is particularly relevant to collective-action traps. Regulatory arrangements seek to alleviate the tragedy of the commons; arms control agreements seek to defuse the momentum of arms races. But institutional adjustments can help to alleviate structural traps as well. Zoning systems can steer patterns of urban and suburban development and channel choices regarding infrastructure needed to accommodate these patterns. Institutional arrangements aimed at improving the education of girls in poor communities can alter demographic and economic forces in ways that can help to alleviate poverty. Nevertheless, institutional interventions are 
hardly a panacea when it comes to escaping from resilient traps. Institutional arrangements that look promising on paper often prove insufficient to control the forces leading to the reemergence of authoritarianism. As those who have struggled to control the spread of firearms through regulatory measures know well, it is not only difficult to get policymakers to agree to meaningful regulations at the outset; it is also extremely difficult in many cases to implement them effectively. On a grander scale, the creation of the United Nations to take action against "threats to the peace, breaches of the peace, and acts of aggression" has not provided any guarantee that we are no longer vulnerable to the Thucydides trap (United Nations 1945). Institutions do play a significant role in efforts to escape social and socioecological traps under some conditions. But they certainly do not provide an all-purpose solution.

This brings us to the role of behavioral tipping elements. Human behavior lies at the heart of social and socioecological traps. Sometimes, the behavior in question involves conscious calculations on the part of those seeking to promote their own interests. Many collective-action traps, for example, arise from the calculations of those engaged in interactive decision-making who seek to maximize relative gains or to protect themselves from the actions of others expected to exhibit such behavior. For their part, structural traps often reflect less conscious efforts of individuals to get ahead or protect themselves in situations over which they have little control. Sometimes, the results are counterproductive, as in many cases featuring the spread of firearms. Often, the results are ineffective, as in many cases involving flight from the perceived ills of urban living. The question that arises in all these cases concerns the extent to which human behavior is malleable. Are we stuck with certain basic features of human behavior baked into our DNA? Or is it possible to influence patterns of behavior associated with the operation of traps through various forms of socialization or the development of cultural constraints? Obviously, it is important to tread carefully in responding to such questions. But there is some basis for optimism in thinking about tipping elements featuring behavioral adjustments. For example, there are human groups in which the dynamic we associate with the tragedy of the commons is a rare occurrence. There are also societies in which levels of trust are high enough to eliminate incentives to purchase firearms based on calculations relating to self-defense.

Finally, it is helpful to introduce a distinction between short-term and long-term solutions to social and socioecological traps. Technological innovations, in particular, often yield solutions that seem remarkable in the short run but that may not prevent the eventual reemergence of resilient traps or the rise of new traps. The development of hatchery fish can prevent stock depletions in the short run. But this intervention alone cannot solve the underlying problem arising from dilemmas of collective action and may lead to negative consequences for affected species. The Green Revolution featuring the use of 
improved crop varieties coupled with the introduction of chemical fertilizers and various forms of pesticides has made possible immense growth in global food production. But it does not provide any guarantee against the eventual return of the Malthusian dilemma (Mann 2019). Today, we are debating the merits of turning to geoengineering as a response to what is increasingly regarded as a climate emergency (National Research Council 2015). But it is already clear that geoengineering is at best a palliative measure. What is more, these technological innovations have a tendency to produce more or less severe side effects. The influx of hatchery fish tends to degrade the vigor of natural stocks. The ecological disruption arising from a reliance on monocrops and liberal uses of chemical fertilizers and pesticides is notorious. Geoengineering provides no solution to the problem of acidification and may prove costly in breeding what is known as moral hazard. None of this is to argue that we should avoid all such interventions, letting nature (including human nature) take its course. But it is clear that we need to bear in mind at all times the problematic consequences that may arise from efforts to take advantage of positive tipping elements that seem to offer hope for breaking out of social and socioecological traps.

\section{TOWARD A BALANCED VIEW OF COMPLEX ADAPTIVE SYSTEMS}

Nothing I have said in this chapter is meant to detract from the significant contributions of mainstream researchers who study resilience. To develop a comprehensive understanding of resilience, however, we need to consider the dark side as well as the bright side of resilience. Social and socioecological traps are common in most societies. In many cases, it is extraordinarily difficult to devise effective escape routes for those ensnared in these traps. And such traps are often highly resilient in the sense that they reemerge in recognizable forms in the wake of disturbances in the form of concerted efforts to break out of them. Cases involving the return of authoritarianism following revolutions and the rise of new threats to the sustainability of living resources following the development of regulatory restrictions provide prominent examples. This does not mean that there are no cases of success in efforts to escape from social and socioecological traps. But it does mean that we must take this problem seriously, seeking to pinpoint the mechanisms involved and devising solutions on a case-by-case basis that are crafted carefully to counteract these mechanisms under real-world conditions.

Finding ways to address two key analytic concerns will be critical to efforts to make progress in developing an empirically grounded and robust theory encompassing both the bright side and the dark side of resilience. One of these concerns has to do with developing operational indicators to distinguish 
between a reorganization within the same system and a critical transition from one system to another distinct system (Scheffer 2009). So long as we are dealing with reorganization within the same system, it makes sense to apply the idea of the adaptive cycle and to argue that the system is resilient. We know what a transition from one system to another or from one basin of attraction to another looks like conceptually (Scheffer et al. 2012). But we lack a straightforward method for applying this concept empirically to identify actual bifurcations. The result in many cases is ambiguity regarding the limits to resilience.

The other concern has to do with treating resilience as a variable. If we could measure variations in levels of resilience with some precision, it would be possible to develop and (at least in principle) test hypotheses about factors or combinations of factors that determine levels of resilience. In the absence of such measures, we often are reduced to treating resilience in dichotomous terms. That is, we are inclined to say that a system is either resilient or it is not resilient. The resultant focus on critical transitions has certainly led to important insights regarding the dynamics of ecological, social, and socioecological systems. But it cuts off a large range of topics that a comprehensive account of resilience should be able to address.

We live in a world of increasingly complex systems marked by hyperconnectivity, nonlinear dynamics, and frequent surprises. Mainstream work on resilience tends to regard this development as worrisome. The focus is on thresholds and tipping points, the maintenance of safe operating spaces, and the importance of applying the precautionary principle to avoid skating on thin ice. While this focus is understandable for those who think about the bright side of resilience, a different reaction to complexity comes into focus when we investigate the prospects for escaping social and socioecological traps. Many traps are hard to escape. That is, they are often resilient in the sense that they show a tendency to reemerge following disturbances or perturbations. In such cases, the operation of teleconnections and the occurrence of bifurcations may present opportunities for breaking out of traps rather than appearing as dangers to be avoided at all costs. Because such developments are apt to take the form of surprises under conditions of complexity, those seeking to escape from traps may be unprepared to recognize these opportunities and to seize them in launching prompt efforts to spring free of the traps. This suggests that there is much to be said for taking steps to sharpen our ability to recognize the onset of bifurcations and to build a capacity to move quickly to take advantage of the opportunities they present. The familiar adage that a severe crisis is too good to waste applies to situations of this sort. But for those seeking to escape traps in complex systems, a necessary condition for success in reacting promptly and effectively to the onset of a crisis will be the development of a capacity 
to recognize the start of critical transitions quickly and to launch vigorous responses without delay. 